Browse $\checkmark$ My Settings $\checkmark$ Help $\checkmark \quad$ Institutional Sign In

Institutional Sign In

All

Conferences > 2020 International Conference.

\section{Development of a Voice Chatbot for Payment Using Amazon Lex Service with Eyowo as the Payment Platform}

Publisher: IEEE

Cite This

PDF
All Authors

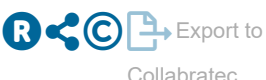

65

Full

Text Views

\section{Collabratec Alerts}

Manage

Content Alerts

Add to

Citation Alerts

\section{Abstract}

Document Sections

I. Introduction

II. Materials and Methods

III. System Implementation

IV. System Implementation

V. Conclusion

Authors

Figures

References

Keywords

Metrics
Abstract:Engaging in financial transactions has remained a hassle for the visually impaired due to the lack of technological products to facilitate their financial independence an... View more

\section{Metadata}

Abstract:

Engaging in financial transactions has remained a hassle for the visually impaired due to the lack of technological products to facilitate their financial independence and inclusion. Automated teller machines (ATMs) and online banking applications do not provide any means through which the blind can engage in transactions without the need of a third party in managing their finances. This study aims at building a voice chatbot device that can be used for payment using Amazon Lex Service with Eyowo as the payment platform. The chatbot is built by leveraging on Artificial Intelligence (Al) technologies in the form of a service called Amazon Lex for configuring the bot with utterances and responses and Lambda Functions to validate the responses while carrying out the transactions by calling the Eyowo Application Programming Interface (API). A Raspberry pi single board computer is utilised as the medium of communication between the user and the chatbot. The Raspberry pi runs a script that collects voice input through a Universal Serial Bus (USB) microphone connected to it, which is sent to the Amazon Lex to be processed using Automatic Speech Recognition (ASR) and Natural Lanquage Understanding (NLU). Then the chatbot sends back a suitable
ADVANCED SEARCH

\section{More Like This}

Voice controlled home automation system using Natural Language Processing (NLP) and Internet of Things (loT)

2017 Third International Conference on Science Technology Engineering \& Management (ICONSTEM)

Published: 2017

Development of GUI for Text-toSpeech Recognition using Natural Language Processing

2018 2nd International Conference on Electronics, Materials Engineering \& Nano-Technology (IEMENTech)

Published: 2018 
Published in: 2020 International Conference on Decision Aid Sciences and Application (DASA)

Date of Conference: 8-9 Nov. 2020

INSPEC Accession Number: 20364463

Date Added to IEEE Xplore: 15 January 2021

DOI: 10.1109/DASA51403.2020.9317214

Publisher: IEEE

ISBN Information:

Conference Location: Sakheer, Bahrain

\section{:三 Contents}

\section{Introduction}

Artificial Intelligence (AI) has brought about some of today's most important technological advances. The emergence in Al and interaction of people with each other and the software used to connect to people has changed the way people communicate by providing an opportunity for people to talk directly to software, thus bringing forth the emergence of chatbots. Chatbots are computer programs that perform human-tomachine conversation in the accomplishment and fulfilment of a task using either auditory or textual interfaces depending on the preferred input modality [1]. Text-based chatbots can perform various tasks ranging from booking a hotel reservation to paying a bill, thus making life easier for consumers [2]. The use of text as the input modality is a limitation since a keyboard is required at every point whenever a user (the blind) wants to communicate to the bot [3]. Voice chatbots are considered more natural and efficient as it does not just make use of the most primary form of communication of humans (speech/voice). Still, it will be providing a more integrated customer experience with immediate verbal feedback, which will allow users to multitask with ease. The use of voice chatbots adds a certain level of trust from the user to the service being used [4], thus increasing the usability and continuous operation of the service to customers [5].

\section{Authors}

Figures

\begin{tabular}{lc}
\hline References & $\checkmark$ \\
\hline Keywords & $\checkmark$ \\
\hline Metrics & $\checkmark$
\end{tabular}

Metrics

\section{IEEE Personal Account}

CHANGE USERNAME/PASSWORD

\section{Purchase Details}

PAYMENT OPTIONS

VIEW PURCHASED DOCUMENTS

\section{Profile Information}

COMMUNICATIONS PREFERENCES

PROFESSION AND EDUCATION

TECHNICAL INTERESTS

\section{Need Help?}

US \& CANADA: +18006784333

WORLDWIDE: +1 7329810060 


$\begin{array}{lll}\text { »Change Username/Password } & \text { »Payment Options } & \text { »Communications Preferences } \\ \text { » Update Address } & \text { »Order History } & \text { »Profession and Education } \\ & \text { »View Purchased Documents } & \text { "Technical Interests }\end{array}$

» US \& Canada: +18006784333

»View Purchased Documents

1) Technical Interests

» Worldwide: +17329810060

»Contact \& Support

About IEEE Xplore $\mid$ Contact Us $\mid$ Help $\mid$ Accessibility | Terms of Use | Nondiscrimination Policy $\mid$ Sitemap | Privacy \& Opting Out of Cookies

A not-for-profit organization, IEEE is the world's largest technical professional organization dedicated to advancing technology for the benefit of humanity.

(c) Copyright 2021 IEEE - All rights reserved. Use of this web site signifies your agreement to the terms and conditions. 